\title{
Botulism outbreak in a family after ingestion of locally produced cheese
}

\begin{abstract}
Botulism is one of the most important foodborne diseases and is caused by Clostridium botulinum toxin. The main manifestations are flaccid muscle paralysis and cranial nerve palsies. Botulism is an essential health problem because of its high mortality. The diagnosis of botulism, especially in sporadic cases, is a medical challenge and a high clinical suspicion is necessary for early recognition. So, every physician should be familiar with its signs and symptoms for early detection and treatment. We describe a family with dysphasia and acute paralysis after the ingestion of locally made cheese. The clinical presentations of the $1^{\text {st }}$ patient were similar to myasthenic crisis and she, therefore, received plasma exchange. After the appearance of similar symptoms in the other family members, they were treated with polyvalent botulinum antitoxin and diagnosis was confirmed by toxicology and detection of serotype Abotulinum toxin in cheese and stool samples. Uncommon clinical presentations and unusual sources of botulinum toxin should be kept in mind because of the importance of early diagnosis and treatment.
\end{abstract}

Volume 6 Issue 5 - 2017

Shahram Rafie,' Shokrollah Salmanzadeh, ${ }^{2}$ Asieh Mehramiri,' Adel Nejati'

'Department of Neurology, Ahvaz Jundishapur University of Medical Sciences, Iran

${ }^{2}$ Department of Infectious Diseases, Ahvaz Jundishapur

University of Medical Sciences, Iran

Correspondence: Asieh Mehramiri, Department of Neurology, Ahvaz Jundishapur University of Medical Sciences, Golestan Hospital, Iran, Tel +98 6II 37430 I2,

Emailmehramiri.ac@gmail.com

Received: April 17, 2017| Published: April 24, 2017

Keywords: botulism, botulinum toxin a, cheese, clostridium botulinu, blurred vision

\section{What's known}

i. The most common positive findings of patients with botulism on examination are ophthalmoplegia, ptosis, and slurred speech. Sparing of extraocular muscles is atypical. Anorexia, nausea and vomiting, and abdominal pain are the most common gastrointestinal complaints with a high prevalence.

ii. In Iran, most outbreaks of botulism have been associated with vegetables and fish; botulism by milk products and cheese is rare.

\section{What's new}

i. Our patients had no gastrointestinal symptoms or signs of ocular involvement, especially pupillary involvement, on physical exam on admission like most patients affected by botulism. Uncommon clinical manifestations should be kept in mind. Also unusual sources of toxins such as home-made cheese should be considered a source of botulinum toxin.

ii. This report is also important given the epidemiological aspects of the disease.

\section{Introduction}

One of the most hazardous diseases, botulism is caused by a toxin produced by Clostridium botulinum, which is a Grampositive anaerobic spore-forming bacterium. It blocks the release of acetylcholine from the motor endplate. ${ }^{1}$ Clostridium botulinum species produce 7 different serotypes of botulinum toxin (A-G). ${ }^{2}$ This disease usually presents as an acute flaccid descending paralysis, combined with visual, bulbar, and gastrointestinal symptoms. Sensory nerves are not affected. ${ }^{3}$ Misdiagnosis is common because of the low prevalence of botulism and its similar manifestations with other neurological diseases like myasthenia gravis. Accordingly, a high clinical suspicion is needed for a correct diagnosis. ${ }^{4}$ On the other hand, early diagnosis, pentavalent botulinum antitoxin treatment (A-
E), and intensive care have reduced the mortality rate from $60 \%$ to below $10 \%{ }^{2}$ Confirmation of the disease rests on the detection of the toxin in the patient's serum, stool, gastric secretions, or food sample. Laboratory data collection is usually delayed, and treatment should be started before the completion of confirmation. ${ }^{1}$

We describe a family (4 members) who were affected by the consumption of contaminated locally made cheese. Because of the importance of the early detection of this potentially lethal disease, we explain the clinical findings and diagnosis of botulism in this family with a view to contributing to its better management in the future.

\section{Case presentation}

On September 23, 2013, a 21-year-old lady (patient 1) from Shushtar referred to Ahvaz Golestan Hospital due to progressive and unremitting blurred vision and dizziness of 5days' duration. Bilateral ptosis and horizontal diplopia forced her to go to a general practitioner, who administered some medications to no avail. After 1-2days, she became dysphagic to solid food, and muscle weakness started from the upper to lower extremities with proximal preference, rendering her unable to walk alone. She had no history of gastrointestinal symptoms such as abdominal pain, vomiting, diarrhea or constipation, fever, excessive sweating, and fluctuation in symptoms, and nor did she have a history of the consumption of any canned food or seafood. On physical examination, the patient was afebrile and had blood pressure of $120 / 80 \mathrm{~mm} \mathrm{Hg}$ with orthostatic change, respiratory rate of $28 / \mathrm{min}$, and pulse rate of $100 / \mathrm{min}$. The pupils were normal sized and reactive to light and had normal directions, gag reflex was reduced bilaterally, and the other cranial nerves were intact. Muscle forces were $4 / 5$ in the upper extremities, $3 / 5$ in the lower extremities, and neck flexor force was $2 / 5$. The patient was dysphagic to solids and liquids and had shortness of breath; accordingly, she was immediately intubated and admitted to the intensive care unit (ICU). Because of her spared pupils and normal accommodation reflex, bulbar sign, and progressive quadriparesis, we started treatment with the impression of myasthenic 
crisis. She received 1 session of plasma exchange (1 plasma volume). The next day, the $2^{\text {nd }}$ member of the family, a 32-year-old lady, was admitted to the emergency room. Her manifestations were the same as the $1^{\text {st }}$ patient but with less severity. Given the appearance of similar symptoms in her family members, our suspicions about botulism grew stronger, so we stopped the plasma exchange. On scrutiny, we found that the patients had consumed contaminated locally made cheese in the preceding week. Stool, blood, and suspected cheese samples were sent for toxicological evaluation to Pasteur Laboratory in Tehran without delay. Two other patients (a 29-year old mananda12-year-old boy) referred during the next 48 hours with identical complaints. Their vital signs on admission were normal, and the main clinical findings were also general muscle weakness and impaired gag reflex with less severity compared to the $1^{\text {st }}$ patient. All of them were admitted to the ICU and treated with polyvalent botulinum antitoxin and conservative management. The result of the toxicological test was positive for botulinum toxin type A. Seven days after admission to the ICU, the family members of our $1^{\text {st }}$ patient were discharged with good general condition, while she herself was discharged 34days after admission to the ICU with mild weakness, which resolved at follow-ups.

\section{Discussion}

Botulism is a rare and potentially lethal, albeit preventable and curable, illness caused by the exotoxin of Clostridium botulinum. Outbreaks of poisoning are most often caused by ingested bacteria contained in home-preserved products rather than by commercially canned products. ${ }^{5}$ Clinical findings usually appear within 12 to 36 hours of the ingestion of the tainted food. Anorexia, nausea, and vomiting (77.4\%); abdominal pain $(54.8 \%)$; and blurred vision and diplopia (48.4\%) are the most common clinical manifestations. ${ }^{6}$ As a rule, blurred vision and diplopia are the initial neural symptoms. The most common positive findings on examination are ophthalmoplegia (79\%), ptosis (76\%), and slurred speech (58\%). ${ }^{7}$ Other manifestations such as bulbar involvement, nasality of voice, hoarseness, dysarthria, and dysphagia follow in quick succession. These, in turn, are followed by the progressive weakness of the muscles of the face, neck, trunk, and limbs as well as by respiratory insufficiency. ${ }^{8}$ Approximately, $68 \%$ of the patients have 3 of 5 symptoms such as nausea and vomiting, dysphagia, diplopia, and dilated and fixed pupils, 7 which are the combination of neurological and gastrointestinal symptoms. Their association with ptosis, strabismus, and extraocularmuscle palsies, particularly of the $6^{\text {th }}$ nerve, may at first suggest a diagnosis of myasthenia gravis. Sparing of the extraocular muscles is atypical in botulism. ${ }^{9}$ Clinical diagnosis can be confirmed by electrophysiological studies and finding of toxin in the patient's serum and stool and suspected food. ${ }^{1}$ Laboratory confirmation takes a considerable time, so clinical findings and response to treatment could be useful in diagnosis. Treatment includes admission of the patient to the ICU and prescription of antitoxins and guanidine hydrochloride. ${ }^{2}$ Antitoxins can neutralize the toxins that are unbound to neuromuscular junctions; consequently, early prescription within the first 24 hours is an ideal treatment. The incubation period can last from 4hours to 10days. ${ }^{3}$ In our patient, blurred vision and diplopia started 4days before admission, followed by progressive weakness of the limbs and respiratory insufficiency. Nevertheless, there were no gastrointestinal symptoms. Additionally, the pupils were reactive at the time of admission and accommodation and extraocular movements were normal too. She did not consume any usual food that might cause botulism. So, the patient was admitted with a diagnosis of myasthenia crisis and was started on plasma exchange. Electromyography/nerve conduction study
(EMG/NCS) is necessary to differentiate between myasthenia gravis and botulism. Decrement on slow RNS are common in botulism so cannot differentiate this disorder from myasthenia gravis. Any increment greater than $40 \%$ is abnormal but incremental response greater than $100 \%$ is suggestive of botulism rather than myasthenia. Electromyography/nerve conduction study was not performed due to the instability of the $1^{\text {st }}$ patient, but the rapid nerve stimulation test and EMG/NCS in the $2^{\text {nd }}$ patient did not show any abnormality or any decremental response. Botulism by home-preserved cheese was first reported10 in New York State in 1914. Although botulism by milk products and cheese is rare, ${ }^{11} 19$ persons in the North of Iran 12 were affected by botulinum toxin type A caused by home-preserved cheese in 1997..$^{10-12}$ In Iran, most outbreaks of botulism have been associated with vegetables and fish. ${ }^{13}$ As much as the incidence of foodborne botulism is low, its frequency is high in middle income countries such as Iran. ${ }^{14}$ Every physician should consider this potentially lethal disease and the uncommon sources of infection for early diagnosis and treatment.

\section{Conclusion}

The aim of this study was to explain our experience regarding the clinical presentations of foodborne botulism in the members of a family. Botulism is still an important public health concern and an epidemiological problem. Although gastrointestinal symptoms in tandem with ocular involvement occur in most patients, their absence cannot exclude botulism. Uncommon clinical manifestations should be kept in mind because of the importance of early diagnosis and treatment. Furthermore, unusual home-preserved foods such as cheese, as was the case in our study, should be considered a source of botulinum toxin.

\section{Acknowledgements}

We thank the family of the patients for allowing us to share their details. Many thanks are also due to Dr. Nader Pazyar for his expertise.

\section{Conflict of interest}

The author declares no conflict of interest.

\section{References}

1. Marcus N, Hourvitz A. Botulism disease. Harefuah Spec No; 2002 p.73-77.

2. Dembek ZF, Smith LA, Rusnak JM. Botulism: cause, effects, diagnosis, clinical and laboratory identification, and treatment modalities. Disaster Med Public Health Prep. 2007;1(2):122-134.

3. Aminzadeh Z, Vahdani P, Mirzaei J. A survey on 80 cases of botulism and its clinical presentations as a public health concern. Arch Clin Infect Dis. 2007;2(2):77-81.

4. Keesey JC. Clinical evaluation and management of myasthenia gravis. Muscle Nerve. 2004;29(4):484-505.

5. Ropper A, Samuels M. Adams and victor's principles of neurology. 9th ed. USA: McGraw Hill Professional; 2009. 1247 p.

6. Rahbar TM, Badsar AR, Akhoundzade N, et al. A survey on the clinical presentations in food-borne botulism for patients refering to Razi Hospital during 2001-2006. Iran J Toxicol. 2012;5:554-557.

7. Varma JK, Katsitadze G, Moiscrafishvili M, et al. Signs and symptoms predictive of death in patients with foodborne botulism-Republic of Georgia, 1980-2002. Clin Infect Dis. 2004;39(3):357-362. 
8. Aureli P, Di Cunto M, Maffei A, et al. An outbreak in Italy of botulism associated with a dessert made with mascarpone cream cheese. Eur $J$ Epidemiol. 2000;16(10):913-918.

9. Gdynia HJ, Huber R, Kastrup A, et al. Atypical botulism sparing palsy of extraocular muscles. Eur J Med Res. 2007;12(7):300-301.

10. Lindstrom M, Korkeala H. Laboratory diagnostics of botulism. Clin Microbiol Rev. 2006;19(2):298-314.

11. Doyle CJ, Gleeson D, Jordan K, et al. Anaerobic sporeformers and their significance with respect to milk and dairy products. Int J Food Microbiol. 2015;197:77-87.
12. Shahcheraghi F, Nobari S, Masoumi Asl H, et al. Identification of botulinumtoxin type in clinical samples and foods in Iran. Arch Iran Med. 2013;16(11):642-646.

13. Barari M, Kalantar E. An outbreak of type Aand B botulism associated with traditional vegetable pickle in Sanandaj. Arch ClinInfect Dis. 2010;5(2):111-112.

14. Habibiyan Nejad Z, Afshari R. Food borne botulism in Mashhad from 2003 to 2010. J Clin Toxicol. 2011;1:115-119. 